\title{
Association between Child Diarrhea and Maternal Depression
}

Milad $\mathrm{G}^{1}$, Izzeldin $\mathrm{S}^{2}$, Tofail $\mathrm{F}^{3}$, Ahmed $\mathrm{T}^{4}$, Hakim $\mathrm{M}^{5}$, Khalil $\mathrm{I}^{6}$, Petri WA

\begin{abstract}
Background: Maternal depression has been found to be associated with increased diarrheal incidence and childhood malnutrition. Objective: The purpose of the present study was to observe whether the SelfReporting Qustinative (SRQ-20) questionnaire was sensitive enough to pick-up the depressive symptoms of mothers in the urban slum community. Methodology: This was a pilot study in a Dhaka Shantytown and women were interviewed to examine the relationship between maternal depression and their children's diarrheal morbidity. In addition to other socio-demographic information, the Self-Reporting Questionnaire (SRQ-20) was used to screen for maternal depression. Result: A total number of 55 women were interviewed to examine fifty-one percent of mothers scored within the high-risk psycho-morbidity group, suggesting depression. High SRQ scores significantly correlated with poor marital relationships (Regression coefficient \pm standard error $=-0.624+0.225, \mathrm{p}=0.008 ; 95 \% \mathrm{CI}$ - $1.076,-0.172)$. High-risk mothers breastfed for a shorter duration than low-risk mothers ( 3.4 vs. 4.4 months, $\mathrm{p}=0.35)$ and their children had more diarrheal episodes $(2$ episodes vs. $1, \mathrm{p}=0.18$ ), although these differences did not show statistical significance. Conclusion: Depression is common among mothers in urban slums and that a well-designed large study is required to further explore the provocative relationship between maternal depression and child diarrhea with subsequent malnutrition to improve the quality of life of those at risk. [J Shaheed Suhrawardy Med Coll, 2013;5(1):14-20]
\end{abstract}

Key words: Maternal depression, Self-Reporting Questionnaire 20, Bangladesh, breastfeeding, infantile diarrhea

Received: November' 2012; Revised: March' 2013; Accpted: May' 2013

\section{Introduction}

Postpartum depression is a common illness that has a deleterious effect on maternal health as well as childcare and health. One of the pathways by which poor maternal health and depression leads to poor child health is inadequate breastfeeding which leads to a weakened immune system and thus, poor protection against infectious illnesses. Exclusive breastfeeding requires good health of a mother to fulfill her role as the source of nutrition for the child ${ }^{1}$. A qualitative systematic review of literature suggests that women with depressive symptoms in the early postpartum period may be at increased risk for negative infant feeding outcomes including decreased breastfeeding duration, increased breastfeeding difficulties and decreased levels of breastfeeding self-efficacy ${ }^{2}$. Similarly, socioeconomic status of a mother and her family has also been shown to have a significant impact on the mother's breastfeeding habits and her child's overall health. Flacking and colleagues have stated that lower maternal education, mothers' receipt of unemployment and social welfare benefits, and lower household income were individually associated with the start of complementary feeding before the age of 6 months; however this is earlier than recommendation of $\mathrm{WHO}^{3}$.

Bangladesh is a developing country and a unique place to study the relationships between maternal and child health. The high poverty rate is assumed to have a significant impact on health, education and well-being of the family unit ${ }^{4}$. Also, the status of women especially among low socioeconomic classes has been shown to be detrimental to maternal wellbeing ${ }^{5}$. All these factors, along with the high diarrheal incidence in Bangladesh ${ }^{6,7}$ points to a potential link between mothers' socioeconomic status, state of depression, and the diarrheal morbidity of their children.

This information led us to look into the possible correlation

1. Grace Milad, University of Virginia School of Medicine, Charlottesville, VA, USA

2. Saria Izzeldin, University of Virginia School of Medicine, Charlottesville, VA, USA

3. Fahmida Tofail PhD, International Centre for Diarrheal Diseases Research (ICDDR, B), Dhaka, Bangladesh

4. Tahmeed Ahmed, PhD, International Centre for Diarrheal Diseases Research (ICDDR, B), Dhaka, Bangladesh

5. Maliha Hakim, Professor \& Head, Department of Neurology, Shaheed Suhrawardy Medical College, Dhaka

6. Ibrahim Khalil, Assistant Professor, Department of Neurology, Shaheed Suhrawardy Medical College, Dhaka

7. William A. Petri, PhD, University of Virginia Health System, Division of Infectious Diseases and International Health, VA, USA

\section{Correspondence}

Fahmida Tofail, MBBS, PhD, Associate Scientist Clinical Scienses Division, ICDDR,B, 68 Shaheed Tajuddin Ahmed Sarani, Mohakhali, Dhaka 1212, Bangladesh, Telephone \#: 00880-2-8860523-32 Ext. 2350, Fax \#: 00880-2-9885657, Email: ftofail@icddrb.org

Conflict of interest: No conflict of interest was declared by authors.

Financial Support: Pfizer Initiative for International Health/University of Virginia Center for Global Health Scholarship, Benjamin Kean Traveling Fellowship from the American Society for Tropical Medicine and Hygiene, and NIH Grant AI043596

Contributions by authors: The hypothesis for the secondary data analyses was developed by GM, SI, FT, TA and WAP. The fieldwork was conducted and supervised by GM and SI by the help of other supporting staff. FT, TA, HM, KI and WAP did the statistical analysis. GM wrote the first draft and coordinated completion of the paper with SI, FT, TA and WAP. FT had full access to all the data in the study and takes responsibility for the integrity of the data and the accuracy of the data analysis. All authors critically commented on and edited earlier drafts and approved the final version of the paper. 
between the mother's mental health and her child's diarrheal status in a developing country setting where poverty is highly prevalent.

This pilot study, as a part of a larger study, attempts to observe whether the SRQ-20 questionnaire is sensitive enough to pick -up the depressive symptoms of mothers in the urban slum community. During adaptation of the questionnaire, we also took the opportunity to see the specific health and demographic indicators of the mother that may affect her ability to properly feed her child and its consequences on the diarrheal incidence in her child. These variables include presence of depression, socioeconomic status, and obstetric history. The purpose of the study was to observe the impact of a mother's mental health on child feeding practices as an indicator of child rearing practices and the diarrheal outcomes of the child.

\section{Methodology}

This was a cross sectional study conducted from July 2009 to December 2009. Mothers were selected from a community in a shantytown of the Mirpur of Dhaka. Most of the women interviewed were housewives and many were also weavers of cloths. Their husbands were generally doing manual labor jobs or were rickshaw wallahs (pullers), or were unemployed. Mothers aged 18-35 years old, who were in apparent good health, and with a child less than 2 years of age residing in a section of Mirpur slum were included in the study. Seventy mothers were identified by female community health workers who keep a running census of the Mirpur site. Using the census list, mothers were approached door-to-door and if interested were read a verbal consent form. Once the mother agreed to participate, they were interviewed about their socio-demographic information, feeding practices and infant morbidity. Afterwards, they were given a time in which they could come to the site office and they were asked about their marital relationship and screened for depressive symptoms. The instrument had questions regarding demographics and brief obstetric history of mother, socioeconomic status, breastfeeding practices and information on child morbidity the Self-Reporting Questionnaire-20 (SRQ-20), questions regarding their relationships with their husband and motherin-law and anthropometry of the children. The instrument was translated from English to Bangla at the ICDDR,B and was made culturally relevant. The entire structured interview was administered by trained psychologists from the ICDDR,B having inter-observer reliability $\mathrm{r}=0.85$ to 0.90 . Mother's age, highest level of education attained by years of schooling, marital status and obstetric information are elicited. A brief account of the living conditions of the mother and her family were taken during the initial visit during recruitment if the mother wished to join the study. Measures included number of people living in the house, size of the house, water supply and treatment (if any), type of sewage system and type of material used in construction of the house. Materials used for house construction were summed to produce a "housing index" based on construction of roof wall and floor of the house. A "crowding index" was also calculated as the number of people living in a home divided by the number of rooms in the house. Breastfeeding practices were noted down by asking the mother if exclusively breastfeeding of the child was done and for how many months. This was to determine the number of mothers who had followed the WHO's recommendation to exclusively breastfeed the first six months and also later to see if there was a correlation between postpartum depression and early weaning ${ }^{8}$. WHO definitions was used to categorize the feeding habits of the mothers as exclusive breastfeeding, predominant breastfeeding, and partial breastfeeding 9 . The mother was also asked to give a short narrative about the breastfeeding experiences including the motivations to breastfeed, knowledge about its benefits, use of pre-lacteals and problems encountered. Diarrheal morbidity was characterized based on duration of an episode, frequency in the past year of the child's life and whether or not the child had to go to a hospital for care and/or get medical treatment. A single diarrheal episode was defined as an episode separated from another by at least 3 diarrhea-free days. The SRQ was designed to be a selfadministered survey. However, since most of the mothers were illiterate, this method of administration was not possible and the questions were read to them. All mothers were also asked to describe their relationships with their husband and mother-in-law using both a Likert scale to a given statement where higher scores indicated better relationship and also by giving an open response. Weights of all the enrolled children were measured by electronic or beam scales, which were precise to $10 \mathrm{~g}$ (UNICEF Uniscale, SECA Gmbh \& Co., Hamburg, Germany). Locally manufactured, collapsible length boards, which were precise to $1 \mathrm{~mm}$, were used to measure recumbent length of the children. Weight and length measurements were converted to weight-for-age (WAZ), length-for-age (LAZ), weight-forlength (WLZ) and BMI-for-age (BMIZ) Z-scores according to the new WHO child growth standards. To assess the clarity and flow of this survey, mock interviews on five mothers from the Mirpur site and the ICDDR, B Nutrition Unit were administered to assess the extent to which the mother is able to understand and answer questions and identify questions and/or translations with a problem. This study was approved by the Ethical Committee of Sir Salimullah Medical College and Ethical and Research Review committees of ICDDR, B. Statistics were done with the SPSS 19.0 (SPSS Inc, Chicago). Initially, descriptive analyses were performed to check distribution of data. All data were checked for normality. Partial correlation controlling for age and sex was conducted to see the relationship of socio-demographic factors with exposure like maternal depression and outcome variables like diarrheal episodes and duration of EBF. Group differences were tested by independent sample t-test and ANOVA for continuous variables and Chi-square test for categorical variables. Finally, in the multiple linear regression analyses, 
all the variables were significantly different between the groups and were correlated with exposure and outcome variables.

\section{Results}

Seventy mothers were invited to participate and 55 gave informed consent. Five of the seventy mothers (7.0\%) refused to participate. This study was conducted during the rainy season and the heavy rains were cited as a primary reason deterring mothers from walking to the office for the latter half of the interview. Ten mothers $(14.0 \%)$ were lost to follow-up. The highest level of education attained for $72.7 \%$ of participants was the completion of primary school. About $74.0 \%$ of the women in the study did not complete secondary school. All of the women were married. Obstetric history showed that more than two third of the women had given birth in their homes; the rest gave birth in a hospital or community clinic. In terms of socioeconomic status (Table $1 \mathrm{~b}$ ), the majority of women lived in households of 3-5 people in a one-bedroom home. Most of the houses were constructed partly with concrete and partly with tin and/or bamboo. Nine mothers (16.4\%) did not treat their water for bacterial contamination. The majority of those who did treat their water did so by boiling. The living conditions were generally the same among all the women interviewed due to the fact that they all lived in the same neighborhood and that each set of four or five homes (single rooms) would share a communal bathroom and stove. Each row of houses had communal water supplies; a minority of the households had private water supplies (called tubewell). Table 1c provides information about breastfeeding in this population: $80.0 \%$ of mothers exclusively breastfed for any given amount of time; $56.8 \%$ breastfed for the ideal 6 months. Only one mother reported that she continued exclusive breastfeeding for 12 months: this was excluded from the analyses as an outlier. Forty-two percent of mothers gave their child honey, sugar or mustard oil before breastfeeding on the day of their birth. The vast majority of mothers said they were satisfied with breastfeeding, noting that it was the most available and inexpensive option- "formula is too expensive" and those they were told "it is good for brain development". Only a few mothers complained they had occasional breast pain. Those that were unsatisfied with breastfeeding explained that they "did not have enough milk" and that their "baby does not latch on my milk." Table $1 \mathrm{~d}$ shows that more than half of the children in the study had at least one episode of diarrhea in the past year: $60 \%$ had more than two episodes in the past year. One mother reported that her three-month old child had persistent diarrhea.

It was suspected that this is not a case of infectious diarrhea but more likely a malabsorption syndrome or enzyme deficiency. The majority of children $(80 \%)$ who experienced diarrhea were not sick enough to get admitted to a hospital but were seen as an outpatient and got medical treatment for it $(93.7 \%)$. Women at high risk for psychometric morbidity were found in a high percentage and $50.9 \%$ of women

\section{Table 1: General Characteristics of the Population}

\begin{tabular}{cc}
\hline Mean \pm SD or percentage (\%) \\
Characteristics
\end{tabular}

\section{1a. Demographic Information of Mother \& Child}

Maternal Age (years)

$24.82+4.20$

Education Level Attained

$72.7 \%$

Child's Age (months)

$13.04 \pm 6.24$

Child Sex (\% male)

$52.7 \%$

1b. Socioeconomic Factors

Crowding Index

Type of Housing Material

$4.19+1.53$

$89.1 \%$

Sewage (\% Sanitary latrine)

$78.2 \%$

1c. Breastfeeding Patterns

Exclusive Breastfeeding in months

(median, interquartile range)

1d. History of Diarrheal Morbidity

Diarrheal incidence (\% yes)

Number of Diarrheal Episodes

(Median, inter-quartile range)

Medical Treatment for Diarrheal Episodes (yes)

1e. Quality of Relationships

Relationship with husband

(with unhappy or unstable relationship)

Relationship with Mother-in-Law

(with unhappy or unstable relationship)

1f. Self-Reported Questionnaire

Low Risk of Psychometric Morbidity

( $\%<7$ scores $)$

SRQ score ( Median, interquartile range)

*Education Level Attained $=\%$ completed primary education * Crowding Index=number of people/no of room

*Type of Housing Material= \% Houses without mud structure

had SRQ-20 scores of 7 and above. More than half (65.5\%) of the women said they were satisfied with their marital relationship while $50.5 \%$ described their relationships with. In the open-response section of these questions, a number of women who described their relationships as "good" or "very good" stated that they were verbally or physically abused by the family member but did not think that warranted a lower score. "Yes, he beats me...but I don't think that's significant," one woman explained.

Table 2: Socio-demographic factors correlated with maternal depression, diarrheal episodes and duration of EBF, controlling for age and sex of child

\begin{tabular}{lccc}
\hline $\begin{array}{l}\text { Socio-demographic } \\
\text { factors }\end{array}$ & $\begin{array}{c}\text { Duration of EBF } \\
\text { (months) }\end{array}$ & $\begin{array}{c}\text { Total SRQ } \\
\text { score }\end{array}$ & $\begin{array}{c}\text { No. of Diarrheal } \\
\text { Episodes }\end{array}$ \\
\hline Relationship with Husband & $\mathrm{NS}$ & $-0.350^{* *}$ & $\mathrm{NS}$ \\
Number of abortions & $\mathrm{NS}$ & $\mathrm{NS}$ & $0.393^{* *}$ \\
Abortions Done & $\mathrm{NS}$ & $\mathrm{NS}$ & $0.294^{*}$ \\
Maternal Chronic Illness & $\mathrm{NS}$ & $\mathrm{NS}$ & $\mathrm{NS}$ \\
Number of children & $\mathrm{NS}$ & $\mathrm{NS}$ & $-0.279^{*}$ \\
Crowding Index & $\mathrm{NS}$ & $\mathrm{NS}$ & $\mathrm{NS}$ \\
Full term Child & $-0.474^{* *}$ & $\mathrm{NS}$ & $0.347^{* *}$ \\
\hline
\end{tabular}

$* \mathrm{p}$ value $=0.05 ; * * \mathrm{p}$ value $=0.01 ; \mathrm{EBF}=$ Exclusive Breastfeeding; Crowding Index $=$ number of people/no of room; *Full term Child=at Delivery $>37$ gestational weeks $($ term $=1 /$ preterm $=2)$; $S R Q=$ Self-Reported Questionnaire 
Table 2 shows partial correlations (controlling for child's age and sex) of different socio-demographic and maternal factors with depression and outcome variables (breast feeding duration and child's diarrheal episodes). Significant correlations were found with diarrheal incidence and exclusive breastfeeding duration. An obstetric history of abortions (irrespective of type or number of abortions) significantly correlated with the number of diarrheal episodes in the child. The more children the woman had, the less number of episodes of diarrhea the child experienced. Additionally, pre-term children were more likely to not have been exclusively breastfed than their full-term counterparts and when they were, it was for a shorter duration. If the mother had a history of a chronic illness, the child was also more likely to have had diarrhea than a child of a healthy mother ( $p$-value $>.05$ ). This study found no significant associations found between maternal depression and the two outcomes like breastfeeding habits and diarrheal incidence.

When analyzing for independent determinants of high SRQ scores, i.e. high risk psychometric morbidity, the quality of the relationship the woman had with her husband was the sole significant factor; the worse the marital relationship was, the higher the SRQ score. Nonetheless, this did not significantly impact on breastfeeding or morbidity outcomes.

When it was grouped the mothers into high (SRQ score > 7) and low (SRQ score $<7$ ) risk psycho-morbid group, it was found that high-risk mothers tended to breastfeed for shorter duration compared to low risk mothers. Similarly, the children of high-risk mothers suffered from more episodes of diarrhea compared to children of low-risk mothers (Figure 1): an average of 1.9 episodes compared to 0.9 episodes ( $\mathrm{p}$-value $>0.05$, non- significant result).

Multiple regression analysis to find independent variables affecting each outcome after controlling all the sociodemographic variables that had significant correlation with both exposure (depression) and outcome variables like exclusive breast feeding and diarrheal episode. Using episodes of diarrhea as the outcome, it was controlled for history of abortion (yes/no), birth order of child, prematurity and for the model using breastfeeding as the outcome, it was controlled for prematurity and exposure to prelacteals. In both models we controlled for relationship of women with their husbands. Table 3 shows that, after controlling for the potential confounders measured, maternal depression had no association with exclusive breastfeeding practices or diarrheal episodes of the child. However, infants' maturity at birth showed independent association with both outcomes and mothers' history of abortion showed independent association with children's episodes of diarrhea only.

\section{Discussion}

The SRQ-20, developed by Harding et $\mathrm{al}^{10}$, adapted from four psychiatric morbidity instruments, is commonly used in developing countries for screening psychiatric disturbances. It taps into multi-dimensional mental illnesses including anxiety, depression and stress ${ }^{11}$. This instrument has been used in other studies in Bangladesh ${ }^{12}$ and a cut-off point of 7 points was used for high-risk psychiatric morbidity ${ }^{13,14}$. The SRQ has been used in many developing countries to screen for maternal depression ${ }^{15}$. In China, the ideal cut-off point has been established to be $6 / 7$ with $93 \%$ sensitivity and $62 \%$ specificity ${ }^{16}$. The SRQ is comparable and in some studies performs better than the Edinburgh Postpartum Depression Scale, another commonly used screening instrument and has been shown to be sensitive in picking up postpartum depression $^{17,18,19}$. WHO stardard ${ }^{20}$ was taken for mesuremmtay WAZ, LAL, WLZ \& BMIZ. The high SRQ scores that were obtained in this study are interesting to note, even though these were not significantly correlated with the mothers' breastfeeding and diarrheal incidence of the child. The percentage of mothers who scored within the high-risk psychomorbidity, particularly depressive symptoms, is similar to the report published from Matlab, a rural district in Bangladesh: $52 \%$ of mothers screened positive for depression in one study ${ }^{21}$. Although it was found the children of highly depressed mothers tended to be exclusively breastfed for shorter time and suffered from more diarrheal episodes (fig 1), thes statistical significancte in these numbers as previous studies have found ${ }^{22,23}$. The fact that who scored high on the SRQ-20 are not necessarily suffering from postpartum depression, but from a milder, chronic form of depression or dysthymia. It may also be due to recall bias of the diarrheal information that was collected.

Although the women were not asked they were capable of caring for their children or had thoughts of hurting their children the most clear examples of postpartum depression ${ }^{24}$ or systematically assessed for their functionality, there was no mention of this in the interviews and no evidence of it during observations in the home and the office. Postpartum depression typically occurs within the first 2 weeks to 6 months after delivery, most symptoms of which resolve within one year ${ }^{25}$. The average age of the children surveyed was 13 months, which is more than 6 months past the typical peak period of postpartum depression. Thus, mothers who may have had PPD may have been missed due to the timeline of the study. A recent study showed that women with prenatal depressive symptoms were roughly twice as likely to formula-feed as breastfeed but depressive symptoms were not associated with failure of initiating breastfeeding ${ }^{26}$. This supports this argument that the depression that the mothers in this study experience is a mild form that when faced with their economic pressures, has no impact on their breastfeeding preferences. It was evident that their impoverished economic environment and the harmful relationships commonly seen with their husbands had the largest toll on their psychometric morbidity. This may be an example of adaptation; mothers often stated that they had no other alternative to breastfeeding because even if they didn't want to breastfeed, they could not afford formula.

Finally, the sample size may have affected the final results. The study likely included mothers who were affected by 
PPD but because the sample size was so small, and the prevalence of postpartum depression is usually around 10$20 \%{ }^{27}$, the anticipated effects of PPD were not seen. Another factor that undermines the verity of assessing depression is that the SRQ was administered orally and not self-administered as it is ideally done due to the illiteracy of the majority of the mothers.

Studies in India and Pakistan with larger sample sizes have found that maternal depression is significantly correlated with diarrheal and nutritional status of the child and have consequently identified maternal depression as a risk factor for diarrheal incidence in children ${ }^{28,29}$. In rural Bangladesh and rural India, studies have also demonstrated that maternal depressive symptoms are correlated with stunting of growth in children ${ }^{30,31}$. Studies in Jamaica, Ethiopia and Peru showed no significant correlation between maternal depression and child malnutrition ${ }^{32,33}$. Results similar to those found in India and Pakistan were expected in this study; explanations to account for the observed differences between this study and those of India and Pakistan has been analyged. In assessing factors that had an effect on high SRQ scores, the quality of the marital relationship was the only statistically significant independent variable (B coefficient $=-0.624+0.225, \mathrm{p}=0.008,95 \% \mathrm{CI}:-1.076-$ 0.172). The worse the relationship was, the higher the woman's SRQ score that is depressive symptoms. Interestingly, the satisfaction rate of the mother's relationships with her husband $(65.5 \%)$ was higher than expected when taking into consideration the reality and stories of some of the mothers. One of the observations that were made during fieldwork was the fact that a woman who was experiencing domestic abuse did not see herself as having a "bad" relationship with her husband. When asked, many of the mothers interviewed stated that their relationships with their husbands were very "stable and happy," giving them a score of 5 out of 5. But when asked if they "cry more than usual," as part of the SRQ, they would respond "Yes, when my husband beats me." Women would say that sometimes they were beaten because "my husband thinks I don't do a good job caring for my child" or "when he has a bad day at work." Although the health of the child has not been shown to be affected by the mother's psychological environment and social pressures in this study, medical professionals have a moral obligation to ask about the safety of the mother and her welfare in every clinical encounter.

The correlations that were found between some of the confounding factors and the outcomes of this study are worthy of note. It was shown in Table 2 that there was a positive correlation between having abortions, the number and the type of abortions, with the number of diarrheal episodes the child experienced within the past year. The majority of the abortions these mothers had were spontaneous abortions, with two being induced abortions. The correlation with type of abortion and diarrheal episodes implies that mothers who went through a more invasive abortion (induced or medical) are more likely to have a child with a higher number of diarrheal incidences. This is an indirect effect of a very small sample that cannot be explained by one rationale. Further, no additional information about the reason for the abortion or its outcomes was documented and so no conclusions can justify the statistical finding. The positive correlation between the presence of maternal chronic illness and number of diarrheal episodes in the child once again points to the idea that if a mother's health is compromised in some way, her ability to carry a child to term, uneventful delivery and ability to provide care and nourishment will reflect on the child's health.

The negative correlation between number of siblings and number of diarrheal episodes suggests that the more experienced a mother is in child rearing, the less likely her child will suffer from diarrhea. The socioeconomic data and its correlation with diarrheal incidence and exclusive breastfeeding habits vary with different factors. The above results show a positive correlation between the number of people living in a home and whether a mother exclusively breastfed her child. This might suggest that a larger household contains more experienced elderly figures who encourage the younger generations to breastfeed their child for "good health and nutrition," a statement that was said by many of the mothers interviewed. This hypothesis, however, cannot be assessed because the data of who is currently living in the house were not collected.

The results also show that preterm children were less likely to be exclusively breastfed and experienced more diarrheal episodes. This may suggest that mothers of preterm children suffered from more morbidities and have insufficient breast milk. The decreased likelihood of being exclusively breastfed exacerbates the inherent risk that preterm infants often experience increased morbidity compared to full term infants.

It was also seen that those children who were not given prelacteals were more likely to have been exclusively breastfed. This may be a reflection of a mother's intent to not supplement breast milk even if it means not following cultural customs.

There were some limitations of the present study. The survey relied heavily on the mothers' recall of breastfeeding habits, obstetric history, and diarrheal history of the child. Given that the mother was the source of all information and that depressed patients are more likely to forget things in the past, under-reporting of diarrheal episodes is a real possibility and would bias the study's results towards the null hypothesis. A study assessing parental recall ability of their children's diarrheal incidences found that the recall ability among informants linked to 33 children, who had diarrhea over the preceding four weeks, less than one-third 
reported the occurrence of diarrhea in their children ${ }^{34}$. Another issue that arose was the definition of a "diarrheal incidence". A concrete definition for the mothers was not provided; rather the mother's impression of what constituted diarrhea was used. To assess the number of episodes, we asked the mothers to differentiate episodes with a 3-day gap in between.

\section{Conclusion}

High prevalence of depressive symptoms is observed among mothers of poor urban slums in Dhaka city when assed by SRQ-20. The instrument is user-friendly and mothers understood the questions well. Maternal depressive symptoms show significant association with disturbed marital relationship. It also show that mothers with high depressive symptoms tend to give less care to their children, reflected by lower rate of exclusive breast-feeding practices and higher rate of diarrheal morbidities in children.

\section{References}

1. Mercer RT. Postpartum: Illness and Acquaintance-attachment Process. Am J Nurs 1977;77(7):1174-1178

2. Dennis, CL and McQueen K. The relationship between Infant-Feeding Outcomes and Postpartum Depression: a Qualitative Systematic Review. Pediatrics 2009; 128 (4): 736-751

3. Flacking R, Nyqvist KH, Ewald U. Effect of Socioeconomic Status on Breastfeeding Duration in Mothers of Preterm and term Infants. Eur J Public Health 2007; 17 (6): 579-584

4. Streatfield PK, Karar ZA. Population Changes for Bangladesh in the Coming Decades. J Health Popul Nutr 2008;26(3):261-72

5. Gausia K, Fisher C, Ali M, Oosthuizen J. Antenatal Depression and Suicidal Ideation Among Rural Bangladeshi Women: a Aommunity-based Study. Arch Women Ment Health 2009; Epub ahead of print, PMID: 19468825

6. Mihrshahi S, Ichikawa N, Shuaib M, Oddy W, Ampon R, Dibley MJ, et al. Prevalence of Exclusive Breastfeeding in Bangladesh and its Associations with Diarrhea and acute Respiratory Infections: results of the Multiple Indicator Cluster Survey 2003. J Health Popul Nutr 2007; 25(2): 195-204

7. Mondal D, Haque R, Sack RB, Kirkpatrick BD, Petri WA Jr. Attribution of Malnutrition to Cause-specific Diarrhea: Evidence from a Prospective Study of Preschool Children in Mirpur, Dhaka, Bangladesh. Am J Trop Med and Hyg 2009; 80(50): 824-826

8. Hasselmann MH, Werneck GL, Silva CV.Symptoms of Postpartum Depression and early Interruption of Exclusive Breastfeeding in the first two months of life. Cad Saude Publica 2008; 24(Suppl 2): S341-352

9. World Health Organization. Division of Child Health and Development. Indicators for assessing breastfeeding practices: report of an informal meeting. 1991. WHO/CDD/SER/91.14. pg. 16

10. Harding TW, de Arango MV, Baltazar J, Climent CE, Ibrahim HH, Ladrido-Ignacio L, et al. Mental Disorders in Primary Health care: a Study of their Frequency and diagnosis in four Developing Countries. Psychol Med 1980; 10(2):231-41

11. Khan NZ, Muslima H, Bhattacharya M, Parvin R, Begum N, Jahan M, et al. Stress in mothers of preterm infants in Bangladesh: associations with family, child and maternal factors and children's neuro-development. Child Care Health Dev 2008; 34(5):657-64

12. Izutsu T, Tsutsumi A, Islam AM, Kato S, Wakai S, Kurita H. Mental health, quality of life and nutritional status of adolescents in Dhaka, Bangladesh: Comparison between an urban slum and a non-slum area. Soc Sci Med 2006; 63(6):1477
13. Mari JJ, Williams P. A comparison of the validity of two psychiatric screening questionnaires (GHQ-12 and SRQ-20) in Brazil, using Relative Operating Characteristic (ROC) analysis. Psychol Med. 1985;15(3):651-59 14. Khan NZ, Muslima H, Bhattacharya M, Parvin R, Begum N, Jahan M, et al. Stress in mothers of preterm infants in Bangladesh: associations with family, child and maternal factors and children's neuro-development. Child Care Health Dev 2008; 34(5):657-64

15. Stewart RC, Kauye F, Umar E, Vokhiwa M, Bunn J, Fitzgerald M,et al. Validation of a Chichewa version of the self-reporting questionnaire (SRQ) as a brief screening measure for maternal depressive disorder in Malawi, Africa. J Affect Disord. 2009; 111(1-3):126-34

16. Chen S, Zhao G, Li L, Wang Y, Chiu H, Caine E. Psychometric properties of the Chinese version of the self reporting questionnaire-20 in community settings. Int J Soc Psychiatry 2009. Epub ahead of print. PMID: 19592444

17. Hanlon C, Medhin G, Alem A, Araya M, Abdulahi A, Hughes M, et al. Detecting perinatal common mental disorders in Ethiopia: validation of the self-reporting questionnaire and Edinburgh Postnatal Depression Scale. J Affect Disorder 2008; 108 (3); 251-62

18. Pollock JI, Manaseki-Holland S, Patel V.Detection of depression in women of child-bearing age in non-Western cultures: a comparison of the Edinburgh Postnatal Depression Scale and the Self-Reporting Questionnaire-20 in Mongolia. J Affect Disord, 2006;92 (2-3): 267-71

19. Rahman A, Iqbal Z, Bunn J, Lovel H, Harrington R. Impact of maternal depression on infant nutritional status and illness: a cohort study. Arch Gen Psychiatry. 2004; 61(9); 946-52

20. WHO Multicentre Growth Reference Study Group. (2006) WHO Child Growth Standards: Methods and development. Geneva: World Health Organization, 2006. [Available at: http://www.who.int/childgrowth/] Accessed June 14, 2010

21. Rahman A, Iqbal Z, Bunn J, Lovel H, Harrington R. Impact of maternal depression on infant nutritional status and illness: a cohort study. Arch Gen Psychiatry. 2004; 61(9); 946-52

22. Rahman A, Bunn J, Lovel H, Creed F. Maternal depression increases infant risk of diarrhoeal illness: a cohort study. Arch Dis Child. 2007: 92(1):24-28

23. Rahman A, Iqbal Z, Bunn J, Lovel H, Harrington R. Impact of maternal depression on infant nutritional status and illness: a cohort study. Arch Gen Psychiatry. 2004; 61(9); 946-52

24. Moore and Jefferson. Mood Disorders. In: Moore, DP, Jefferson JW, editors. Handbook of medical psychiatry. 2nd ed. Philadelphia, PA: Mosby Elsevier, 2004

25. Pearlstein T, Howard M, Salisbury A, Zlotnick C. Postpartum depression. Am J Obstet Gynecol. 2009; 200(4): 357-64

26. Rahman A, Bunn J, Lovel H, Creed F. Maternal depression increases infant risk of diarrhoeal illness: a cohort study. Arch Dis Child 2007: 92(1):24-28

27. Gabbe SG. Mood Disorders. In: Gabbe SG, Niebyl JR, Simpson JL, editors. Obstetrics: normal and problem pregnancies. 5th Edition. Philadelphia, PA: Churchill Livingstone, 2007

28. Rahman A, Iqbal Z, Bunn J, Lovel H, Harrington R. Impact of maternal depression on infant nutritional status and illness: a cohort study. Arch Gen Psychiatry 2004; 61(9): 946-52

29. Anoop S, Saravanan B, Joseph A, Cherian A, Jacob KS. Maternal depression and low maternal intelligence as risk factors for malnutrition in children: a community-based case control study from South India. Arch Dis Child 2004; 89(4): 325-29

30. Black MM, Baqui AH, Zaman K, El Arifeen S, Black RE. Maternal Depressive symptoms and infant growth in rural Bangladesh. Am J Clin Nutr 2009; 89(Suppl): S951-57

31. Anoop S, Saravanan B, Joseph A, Cherian A, Jacob KS. Maternal depression and low maternal intelligence as risk factors for malnutrition in children: a community-based case control study from South India. Arch Dis Child 2004; 89(4): 325-29

32. Stewart RC. Maternal depression and infant growth: a review of recent 
evidence. Matern Child Notre 2007; 3(2): 94-107

33. Harpham T, Huttly S, De Silva MJ, Abramsky T. Maternal mental health and child nutritional status in four developing countries.

J Epidemiol Community Health 2005;59(12) 59:1060-64
34. Melo MC, Taddei JA, Diniz-Santos DR, May DS, Carneiro NB, Silva LR. Incidence of diarrhea: poor parental recall ability. Braz J Infect Dis 2007;11(6): 571-79. 